Maternidad adolescente, entre la escolarización y el fracaso escolar

Teenage motherhood among schooling and school failure

\author{
Volumen 17, Número 1 \\ Enero-Abril \\ pp. 1-22
}

Este número se publicó el $1^{\circ}$ de enero de 2017

DOI: http://dx.doi.org/10.15517/aie.v17i1.27292

Julia Ester Cubillos Romo

Revista indizada en REDALYC, $\underline{\text { SCIELO }}$

Revista distribuida en las bases de datos:

LATINDEX, DOAJ, REDIB, IRESIE, CLASE, DIALNET, SHERPA/ROMEO, QUALIS-CAPES, MIAR

Revista registrada en los directorios:

ULRICH'S, REDIE, RINACE, OEI, MAESTROTECA, PREAL, CLACSO 


\title{
Maternidad adolescente, entre la escolarización y el fracaso escolar
}

Teenage motherhood among schooling and school failure

\section{Julia Ester Cubillos Romo ${ }^{1}$}

\begin{abstract}
Resumen: Este artículo recoge las principales conclusiones de un estudio sobre la trayectoria educativa de mujeres adolescentes que enfrentan el desafío de ser madres. Se trató de un estudio cualitativo cuyo objetivo fue comprender la vivencia de madres adolescentes, para identificar dimensiones que se asocian al retiro y la retención escolar, así como describir las demandas de apoyo para compatibilizar ambos roles (estudiante y madre). Con base en diez entrevistas, realizadas a madres adolescentes escolarizadas en establecimientos municipalizados y madres que no continuaron sus estudios secundarios, se desarrolló un análisis comparativo entre ambas experiencias y sistema de creencias. Se concluye que la maternidad no es un factor que genere de forma causal la deserción escolar; pero sí influye sobremanera las dinámicas de inclusión al interior de los establecimientos educativos, lo cual genera estrés y limita procesos de aprendizaje. Además, la vivencia precoz de la maternidad debilita el capital social, las redes de apoyo y las relaciones interpersonales con docentes y pares. De igual forma, se concluye que la maternidad adolescente incide significativamente en la reflexión sobre los proyectos de vida. Para promover la permanencia en el sistema escolar, es preciso abordar los prejuicios y discriminaciones de género que se dan en su interior, tanto por parte del cuerpo docente como de estudiantes, así como instalar prácticas de cooperación que favorezcan la inclusión escolar.
\end{abstract}

Palabras clave: adolescentes, maternidad, fracaso escolar, escolaridad.

Abstract: This article gathers the principal conclusions of a study on the educational path of teen women who face the challenge of being mothers. This qualitative study aims to understand the experience of adolescent mothers for identifying dimensions that are associated to the retirement and the school retention, also to describe the demands of support to make compatible both roles (student and mother). On the basis of ten depth interviews realized to adolescent mothers enrolled in public school establishments and mothers who did not continue his secondary studies, a comparative analysis was realized between both experiences and system of beliefs. One conclusion is that the maternity is not a factor that generates of causal form the school desertion, it influences of exceedingly the dynamics of incorporation to the interior of the educational establishments, question that also affects in the teen mothers who are kept enrolled in school, generating stress, limiting a complete learning process. In addition, the precocious experience of the maternity debilitates the share capital, the networks of support, and the interpersonal relations with teachers and couples. Also one concludes that the teen maternity affects significantly in the reflection on the projects of life. To promote permanency in the school system must eliminate gender bias and discrimination that occur inside by both faculty and students, as well as cooperation install practices that favor school inclusion.

Key words: teens, motherhood, school failure, schooling.

\footnotetext{
${ }^{1}$ Trabaja en Universidad de Aysén en Chile. Socióloga (Universidad de Chile) y magíster en Educación Emocional (Universidad Academia de Humanismo Cristiano, Chile).
}

Dirección electrónica: juliacubillosromo@gmail.com

Artículo recibido: 29 de setiembre, 2015

Enviado a corrección: 21 de julio, 2016

Aprobado: 21 de noviembre, 2016 


\section{Introducción}

Considerando datos del Instituto Nacional de Estadísticas para el 2010, la población adolescente (10-19 años) de Chile asciende a 2817 251, lo que corresponde al $17 \%$ de la población total.

La fecundidad mirada por grupo de edad se mantiene constante, siendo importante la participación del grupo de 15 a 19 años. En palabras de Rodríguez (2005), se aprecia una "resistencia al descenso de la fecundidad adolescente, ya que la única tasa específica de fecundidad que no cayó abruptamente entre 1960 y 2001 fue la tasa del grupo de 15 a 19 años, que tendió a permanecer constante, con algunas oscilaciones" (p. 130). Para el 2005, el 15,62 \% de las adolescentes es madre, cifra que baja levemente a 15,56 para el 2010 (Minsal, 2012).

Para el 2011, el 50,4 \% de las jóvenes de 15 a 19 años presenta hijos nacidos vivos (Minsal, 2011). Ello es coherente con los datos de la 6 ${ }^{\mathbf{a}}$ Encuesta Nacional de la Juventud, en la cual el $48 \%$ de adolescentes de 15 a 19 años declara haber iniciado su vida sexual. La edad promedio de inicio es 16,4 años en los hombres y 17,1 años en las mujeres, cuestión que disminuye con el tiempo, ya que en 1997 mostraban una edad promedio de inicio de relaciones sexuales de 18 años (INJUV, 2010).

El embarazo y la materno/paternidad en adolescentes se da en un contexto de segmentación social. Según Sadler y Aguayo (2007), la gestación adolescente se ha urbanizado y se ha incrementado en familias que viven en situación de pobreza. A ello se suma la diferenciación educativa, donde un alto porcentaje de niños y niñas con alto nivel socioeconómico asisten a establecimientos particulares, y una alta proporción de bajo nivel socioeconómico asisten a municipalizados. En el caso de los jóvenes, según la Encuesta de Juventud, esta señala que, a medida que se baja de estrato socioeconómico, mayor es el porcentaje de quienes declaran tener hijos.

Hamel (1992) sostiene que "la adolescente embarazada y su hijo corren mayores riesgos de morbilidad y mortalidad comparada con grupos de mujeres de 20 y 35 años, especialmente si la adolescente tiene menos de 16 años de edad" (p. 19); además se observa un menor crecimiento estatural y un déficit nutricional mayor en aquellos hijos de madres adultas. Sadler y Aguayo (2007) advierte que

la edad de la madre no es determinante en la ocurrencia de morbilidad en los lactantes, sin embargo, los hijos/as de adolescentes casadas tienen mayor riesgo de enfermar que los hijos/as de adolescentes solteras, lo que nos indicaría que las distintas 
dinámicas familiares y probablemente el rol que cumplen los abuelos/as son de suma importancia en el estudio de la vulnerabilidad de este grupo de riesgo. (p. 22)

En una proporción significativa de casos, se trata de "embarazos inoportunos, a veces no deseados, y una gran parte de ellos no tendría una familia completa al nacer" (Assef, 1996, p. 5). Esto podría provocar consecuencias socio afectivas en los niños, debido a impactos en la autoestima de la joven madre, producto del "alto nivel tensional podría sobrepasar a la madre adolescente y descargar todas sus frustraciones y conflictos en forma de violencia contra el niño, ya sea en forma física o psicológica” (Assef, 1996, p. 6). Reflexiones de la CEPAL respecto al círculo intergeneracional de la pobreza, advierten que el embarazo adolescente, y la maternidad/paternidad temprana influyen en dicho círculo, ya que los hijos de las madres adolescentes también presentan situaciones que limitan su pleno desarrollo (Arriagada, 2003; Cepal, 2008).

Gutiérrez y Duarte (2005) sostienen que los embarazos en mujeres jóvenes no constituyen un riesgo en sí mismo, dependen de condiciones físico-biológicas, situación socioeconómica, aspectos y bienestar psicológico, "cada contexto específico definirá las condiciones que esa futura madre posee para sí misma y para su bebé tanto durante el embarazo como en el parto, la lactancia y en el crecimiento posterior" (p. 77).

Con respecto a las madres, se observa que disminuye la tendencia a contraer matrimonio; además, mientras más temprana es la maternidad, es mayor la probabilidad de permanecer en estado de soltería, por lo que tienden a vivir con sus familias (Rodríguez, 2005). De igual forma, en este grupo de edad (adolescentes de 14 a 19 años), se observa con mayor frecuencia que el embarazo es producto de abusos sexuales (Molina, Molina y González, 2007). Un estudio de Palma y Quilodrán (1992) revela que dentro de los riesgos para el hijo o la hija de la madre adolescente, se puede presentar el síndrome del fracaso, esto es:

un conjunto de efectos negativos que el embarazo indeseado produce en las distintas esferas de la vida de las adolescentes, especialmente en las menores, entre los cuales se incluye la imposibilidad de completar las funciones de la adolescencia, abandono de la actividad escolar por vergüenza o prohibición, grandes dificultades para rearticular su vida sexual y limitar su fecundidad, imposibilidad de establecer una familia y una vocación y poder cubrir las necesidades propias y las del hijo y asegurar que éste alcance su potencial vital. (p. 3) 
Pese a la mayor vulnerabilidad, una proporción de adolescentes que asume tempranamente la maternidad y paternidad permanece escolarizada. Según el Estudio de la Situación de Maternidad y Paternidad en el Sistema Educacional Chileno (Olavarría, Palma, Donoso, Valdés y Olivera, 2007), realizado con datos del Ministerio de Educación de Chile correspondientes al 2005, el total de estudiantes adolescentes padres y madres (hasta 19 años) matriculados fue de 20.905 estudiantes. De ellos, 78,5 \% corresponde a mujeres y $21,5 \%$ a hombres, el rango de edad donde suele concentrarse la maternidad-paternidad oscila entre 16 a 18 años, la gran mayoría (96,7 \%) tiene un solo hijo, y se concentra en áreas urbanas $(95,8 \%)$.

\section{Metodología}

Fue interés del estudio abordar la inquietud con respecto a qué puede ocurrir con el proceso educativo de adolescentes que enfrentan el desafío de ser madres. La tendencia es pensar que el camino directo es el abandono escolar. Por ello, se buscó comprender su opción por la maternidad y su desenlace, ya sea de permanencia o abandono del sistema escolar, para lo cual las preguntas que intentó responder este estudio fueron: ¿cómo incide en el proceso educativo la maternidad adolescente? y ¿por qué podría darse la maternidad adolescente?

El objetivo general fue comprender la vivencia de maternidad de adolescentes de 14 a 19 años, tanto para aquellas que se mantienen escolarizadas como para quienes no continúan estudios secundarios, para identificar dimensiones que se asocian al retiro y la retención escolar; así como describir las demandas de apoyo para compatibilizar ambos roles (estudiante y madre) que presentan para completar el proceso educativo.

En coherencia con ello, se trató de un estudio cualitativo que priorizó por comprender la perspectiva de los sujetos. Contó con una muestra de 10 casos, en proporción similar de madres adolescentes que no continuaron estudios secundarios y madres adolescentes escolarizadas. Se realizaron entrevistas a profundidad, las que contaron con consentimiento informado y otras en las cuales se resguardó la identidad a través de la confidencialidad.

Se procedió a un análisis de discurso (Canales 2006, Mella 2003), identificando, por cada dimensión explorada, aspectos comunes y aspectos específicos que marcan diferencias entre los casos. Se organizó el corpus de la información con citas ilustrativas extraídas de las entrevistas transcritas, las que se encuentran en el informe de resultados. Una vez realizado el ordenamiento de los hallazgos, se discutieron los hallazgos con 
diversos actores ${ }^{2}$ para realizar el análisis en diálogo con distintos enfoques teóricos, para contribuir con conclusiones más robustas, con la intención de dar recomendaciones para políticas educativas.

\section{Análisis}

Se tomó como referente interpretativo la teoría de campo y habitus de Bourdieu (2000) porque aporta los siguientes elementos: en primer lugar, la interconexión entre estructuras subjetivas y estructuras objetivas (o, dicho de otro modo, estructuras cognitivas y condiciones sociales en las que ellas tienen lugar). Como señala García (2000), citando a Bourdieu (quien se autodenomina como estructuralismo genético o constructivista, con este enfoque):

el análisis de las estructuras objetivas -las de los diferentes campos- es inseparable del análisis de la génesis en el seno de los individuos biológicos de estructuras mentales que son por una parte el producto de la incorporación de las estructuras sociales y del análisis de la génesis de esas estructuras sociales: el espacio social, y los grupos que en él se distribuyen, son el producto de luchas históricas (en las cuáles los agentes se implican en función de su posición en el espacio social y de las estructuras mentales a través de las cuales aprehenden ese espacio). (p. 12)

En esa línea, para buscar responder cómo incide en el proceso educativo la maternidad adolescente y ¿por qué podría darse la maternidad adolescente, se debe comprender las estructuras mentales de los sujetos. En este sentido, la noción de habitus emerge como concepto que sirve para superar la oposición entre 'objetivismo' y 'subjetivismo':

Las teorías 'objetivistas' explicarían las prácticas sociales como determinadas por la estructura social: los sujetos no tendrían aquí ningún papel: serían meros soportes de la estructura de relaciones en que se hallan. A su vez, las teorías 'subjetivistas' tomarían el camino contrario: explicarían las acciones como agregación de las acciones individuales. (Reyes, s.f., párr. 1)

\footnotetext{
${ }^{2}$ Este estudio forma parte de la tesis para optar al grado de socióloga. La discusión y reflexión permanente se dio a través del dialogo con los académicos Fabiola Maldonado y Raúl Urzúa, además con un grupo de tesistas que también fueron becados para realizar sus estudios por el Centro de Análisis de Políticas Públicas en el marco de un proyecto de esta institución con la Fundación Ford.
} 
Desde un análisis lineal convencional, sería muy atractivo concluir: a mayor pobreza, mayor embarazo adolescente; es decir, debido a que se trata de adolescentes de familias que viven o han vivido situación de pobreza, ergo, la estructura social las condiciona y lo obvio es que se embaracen joven, deserten del sistema escolar, priven a sus hijos de un ambiente para un desarrollo pleno y por consiguiente reproduzcan la situación de pobreza heredada familiarmente. Sin embargo, tan atractiva conclusión carece de la mirada de los propios actores involucrados y, con ello, elementos históricos, geográficos, y/o culturales, entre otros, que fisuran posibles análisis lineales (causa- efecto) e instalan complejidades y contradicciones necesarias de comprender si se busca desarrollar políticas y programas de apoyo a las madres adolescentes.

También sería interesante suponer que solo es un problema psicológico; es decir, quienes tienen cierta estructura de personalidad o incluso quienes poseen ciertas patologías son más proclives a embarazarse o el impacto psicológico es tan gran grande que las adolescentes que quedan embarazadas desertan casi automáticamente. Sin embargo, para que ocurra embarazo debe haber otro, no solo la mujer; se trata de una pareja, de una relación, de la noción del cuerpo, de sexualidad, de familia, de nociones que tienen una carga social y cultural; en suma, de estructuras mentales situadas en un espacio/tiempo determinado.

La noción de habitus, vinculada con la de campos, permite no quedarse en ninguna de las dos caras de la moneda (objetivismo - subjetivismo) y ver el fenómeno desde una perspectiva multidimensional y multidisciplinaria. En este sentido, ver las estructuras mentales internas, o habitus (entendidos como esquemas de percepción, pensamiento y acción), así como las estructuras externas o campos de posiciones sociales, permitirá, o al menos esa es la expectativa del estudio, y el llamado a los lectores de este, a aportar una mirada más comprensiva. A continuación, algunas expresiones de esa multidimensional.

\subsection{Habitus y capital cultural: la importancia de los referentes familiares}

La conclusión fundamental de este estudio es que la maternidad no es por sí mismo un factor que propicie, de forma causal y directa, el fracaso escolar. Hay otros factores concomitantes, latentes que, al yuxtaponerse con este cambio biográfico significativo, pueden generar como consecuencia el abandono escolar.

Las adolescentes entrevistadas comparten ciertas características que dan cuenta de un habitus común y que ejerce influencia en lo que a embarazo adolescente se refiere: 
- Ios referentes familiares de maternidad adolescente,

- la valoración a la constitución de familia,

- el débil uso de anticonceptivos.

No obstante, al verse enfrentados a campos diferentes, los elementos distintivos de sus habitus, en particular los que dicen tener relación con el aspecto educativo del capital cultural, se activan y propician diferentes respuestas frente a la permanencia en el sistema escolar.

Asumiendo la noción de habitus como base fundamental para estructurar el análisis, es posible señalar la decisión con respecto a la maternidad y, posteriormente, según la continuidad o quiebre con el proceso escolar, se vincula al habitus de las madres adolescente, transmitido por sus familias, en al menos dos sentidos: rol parental y escolaridad.

Bourdieu (2000) aborda la noción de capital cultural como dimensión que explica el rendimiento escolar y hace una ruptura con otros enfoques que ven la relación con base en "capacidades" - "éxito escolar". En relación con la teoría del capital humano, señala

esta definición pasa por alto, entre otras cosas, el hecho de que el rendimiento escolar de la acción educativa depende del capital cultural previamente invertido por la familia; y olvida además que el rendimiento social y económico de la titulación académica depende del capital social, igualmente heredado, que puede ser movilizado para respaldarla. (p. 138)

La noción de habitus de este teórico "incluye las estructuras mentales o cognitivas mediante las cuales las personas manejan el mundo social. (...) "Un habitus se adquiere como resultado de la ocupación duradera de una posición dentro del mundo social" (Ritzer, 1993, p. 502). En este sentido, las familias juegan un papel fundamental, en tanto estructuras primitivas en la transmisión de elementos que lo constituyen. En otras palabras, para el caso de las madres adolescentes (sean estas escolares o no), son fuertemente influidas por el capital cultural de las familias; en términos de roles parentales:

a) Por un lado, existe en las familias algún familiar significativo que ha sido madre a temprana edad. En la mayor parte de los casos, se trata de la propia madre de las entrevistadas u otra (como tías, primas, etc.), que, al sobrellevar un embarazo y una maternidad a temprana edad, se constituyen como un referente o modelo de referencia 
(como lo" normal"), al momento de examinar la propia situación. Esto no quiere decir que, existiendo este referente, ocurre inmediatamente un embarazo en las adolescentes, porque, de hecho, las entrevistadas manifiestan no haberlo planificado, sino sentir un conflicto durante el proceso de compartir la noticia con los más próximos, especialmente por defraudar a otros y limitar aspiraciones o estilos de vidas que sus familias habían construido como un horizonte deseable (especialmente vinculado a compartir con pares, seguir estudios, trabajar mucho tiempo después, no formalizar vínculos de pareja, etc.).

b) Por otro lado, el haber desarrollado prácticas de apoyo doméstico fue perfilando el rol de mujer - madre en el seno del hogar. Esto es especialmente significativo cuando las adolescentes han sido cuidadoras, pues desarrollan prácticas, imágenes y aprendizajes en torno al cuidado de niños pequeños; así como expectativas con respecto a su proyecto de vida en el cual un eje fundamental es la constitución de su propio núcleo familiar.

En relación con el capital cultural de la familia, específicamente vinculado a la educación, se observa escolaridad completa de los hermanos mayores, hermanos menores estudiando, presencia de algunos recursos educativos en la familia. Antecedentes que hablan de una valoración social a la educación y además de acciones para que las generaciones más jóvenes del núcleo familiar incrementen su capital educativo formal, logrando credenciales (graduación).

Una diferencia entre las madres que continúan estudios y aquellas que no lo hacen es que las primeras tienen referentes significativos con capital cultural formalizado (padres con escolaridad completa, y/o con algún miembro cercano con escolaridad completa); mientras que aquellas que no continúan estudios secundarios presentan padres con escolaridad incompleta. A nivel de las estructuras subjetivas, esta presencia de personas significativas con educación completa e incluso con educación superior marca modelos o referentes por seguir, pilares por los cuales se puede orientar el "deber ser" o lo socialmente deseable que genera reconocimiento familiar.

Estos temas dan cuenta de factores que actúan en el proceso de socialización aportando activamente en la acumulación de capital (Tenti, 2000). Bourdieu (2000) nos señala:

por una parte, es bien sabido que el proceso de apropiación del capital cultural objetivado (o sea: el tiempo necesario para este proceso) depende en primer lugar, del 
capital cultural previamente incorporado en toda la familia. Por otra parte, también sabemos que la acumulación de capital cultural desde la más temprana infancia presupuesto de una apropiación rápida y sin esfuerzo de toda suerte de capacidades útiles- sólo tiene lugar, sin demora ni pérdida de tiempo, en aquellas familias poseedoras de un capital cultural tan sólido que hace que todo el periodo de socialización lo sea a su vez de acumulación. De donde sigue que la transmisión de capital cultural es sin duda la forma mejor disimulada de transmisión hereditaria de capital. (p. 143)

Finalmente, es necesario señalar que las entrevistadas de este estudio, que ven su trayectoria escolar interrumpida, presentan limitaciones para argumentar y describir sus puntos de vista durante las entrevistas, ello no solo queda reflejado en el poco vocabulario verbal, sino también en las respuestas vagas y acotadas. Cabe destacar, sin embargo, que el lenguaje no verbal, expresado tanto en gestos como en entonación de la voz, sí estuvo presente. Una posible interpretación a esta cuestión puede ser la del uso de un código restringido, cuestión que se pone de manifiesto con ausencia o limitaciones de lenguaje para describir la realidad (Berstein, 1974), como también por el bajo desarrollo de competencias emocionales que permitan reconocer sus propias emociones, expectativas, para autoexplicarse la experiencia de vida (Casassus, 2006).

\subsection{Maternidad adolescente y debilitamiento del capital cultural}

Si bien la maternidad no deviene en todos los casos en la interrupción de la trayectoria educativa, sí mella el proceso educativo de las adolescentes. Las entrevistadas que optan por continuar sus estudios hablan de que la compatibilización entre los dos roles resulta en una tensión permanente que, entre otras cosas, impacta en el proceso educativo. Al respecto señalan que su prioridad es el bienestar del hijo o hija, por lo que, en términos prácticos, pese a su voluntad por seguir estudiando, acontecen situaciones de:

- Retraso en el cumplimiento de las actividades escolares;

- Incremento de la inasistencia escolar;

- Descenso de las notas;

- Cambio en el ritmo de estudio;

- Incorporación del hijo o la hija en las actividades de estudio;

- Incremento de actividades y labores domésticas; 
- Disminución de las horas de descanso;

- No hacen referencia al tiempo de ocio y recreación, por lo que se deduce que este también es limitado.

Sobre esto, Bourdieu (2000) nos advierte que el capital cultural se internaliza en al menos tres estados: incorporado, objetivado e institucionalizado, siendo estos, de alguna manera, incrementales. Señala que el primer y más básico estadio supone un proceso de enseñanza y de aprendizaje para la interiorización. En este sentido, es plausible poner en duda el proceso real de acumulación de capital cultural en las madres escolarizadas, con todas las interferencias que este tiene al tratar de compatibilizar las exigencias del rol maternal con el de estudiante, por consiguiente, el proceso formal de aprendizaje se ve mermado.

El capital cultural "incorporado" en un habitus se da cuando se pasa de tener acceso a ciertos elementos culturales (conocimiento, información), a apropiárselos por parte del sujeto. Por consiguiente, se estaría potenciando la asistencia escolar, la participación en un sistema que otorga credenciales (capital social institucionalizado), pero no necesariamente que desarrolle competencias en las madres adolescentes, del mismo modo que sus pares escolares, pese a que las estas puedan tener mayores atributos $y$, como se ha visto en las entrevistas, en ocasiones trayectorias escolares fortalecidas (pre-escolar, buenas notas).

Para atender a las posibles diferencias de apropiación cultural y pensando en estrategias de intervención con las madres adolescentes, es necesario considerar al menos dos elementos:

i) Momento cuando comienza el proceso de apropiación de capital y

ii) Periodo prolongado de apropiación.

Por tanto, intervenciones de acción positiva debieran revertir la limitación en cantidad y calidad de tiempo dedicado al estudio; por ejemplo, al estilo de cursos remediales o de nivelación, o la integración en procesos educativos estimulantes, así como también estrategias para manejar el estrés producido por el proceso de enseñanza bajo el contexto de la maternidad, y mayor autocontrol (lo que incluye hábitos de estudios y sentido de autoeficacia escolar). Estos elementos no solo son pertinentes para el caso de las madres adolescentes, sino también para evitar el fracaso escolar en general (Arguedas y Jiménez, 2007), pero cobran mayor relevancia considerando además las concepciones de genero 
prevalecientes en una sociedad patriarcal, donde se espera que el rol de la mujer - madre sea en el espacio privado, enjuiciando la participación en el espacio público (escuela) en aquellas que, además de mujer - madre, desean ser estudiantes (Alvarado, 2005; Araya, 2004; Castillo y Gamboa, 2013).

\subsection{Maternidad adolescente, proyecto educativo y proyecto de vida}

Formar un proyecto de vida, recogiendo la propuesta de Touraine (2006) cuando se refiere a la escuela del sujeto, se asocia a la constitución de sujeto, que no es sino la búsqueda emprendida por el individuo, de las condiciones que le permitan ser actor de su propia historia. El sujeto es el deseo del individuo de ser un actor, afirmando su individualidad. Desde este punto de vista, podríamos decir que las madres adolescentes enfrentan el desafío de delinear un proyecto, una aspiración de sí, sobre la base del conocimiento propio, de la configuración de expectativas, de las relaciones con otros, de la significación e internalización del entorno, entre otros aspectos.

Este estudio subraya la idea de que la maternidad adolescente marca un antes y un después en la biografía de la joven. En ocasiones, es el hito que re-define el después, ya que estructura un proyecto de vida con metas y objetivos claros, en un corto y largo plazo. Ser padres/madres para algunos(as) constituye en sí mismo una realización personal, para otros(as), especialmente quienes han valorizado la educación formal, les resulta más difícil abordar la paternidad/maternidad (Núñez, Segall y Vivanco, 2002; Sadler y Aguayo, 2007).

Considerando los hallazgos de este estudio, se postula la presencia al menos de tres tipos de situaciones asociadas al proyecto de vida:

a) Sin proyecto de vida claro. Solo en el caso de una de las entrevistadas, que interrumpió su trayectoria educativa, se está en presencia descarnada de esta situación. Se trata de quien vive con su pareja en una mediagua y, según fuentes que derivaron el caso para la entrevista, la pareja presenta dependencia al consumo de sustancias toxicas. Este caso podría reflejar lo que Martínez y Palacios (1996) señalan como desesperanza aprendida, entendida como internalización de negaciones y frustraciones.

b) Proyecto educativo - proyecto de vida. Para algunas de las entrevistadas, su proyección personal va de la mano con completar estudios secundarios e incluso de continuar estudios superiores. En algunos casos, la maternidad refuerza esta idea o contribuye a que se genere dicha proyección, y cuando dicho proyecto educativo ya 
existía, se fortalece con la maternidad, debido a la asunción de responsabilidad por la vida de otro afectivamente significativo. En las madres adolescentes entrevistadas que continúan sus estudios, se observa, al momento de figurarse un futuro próximo, una amplia gama de imágenes de sí mismas y de su hijo, como: "que sea sano, que le vaya bien en el colegio, que tenga una educación de mejor calidad que la mía, que tengamos nuestra casa propia, que yo este titulado", etc. En todas ellas, se instaura un sujeto con voluntad y capacidad de construir un futuro. Esa voluntad está fuertemente relacionada con la actuación presente (continuar estudios), así como a valoraciones sobre el porqué estudiar. A este respecto, y en contrapunto con las madres que interrumpen su trayectoria escolar, las entrevistadas señalan que completar los estudios permite completar una expectativa familiar (e incluso proyección), salir con un grado académico que facilite el trabajo (para el caso de las entrevistadas que se cambiaron a un liceo técnico profesional producto de la maternidad) o el acceso a la universidad (respuesta de quienes estudian en la modalidad científico - humanista).

c) Proyecto familiar - proyecto de vida. Es plausible suponer que, en algunos casos, la maternidad adolescente se explicaría por un deseo consciente o inconsciente de asumir nuevos roles, en búsqueda de mayor autonomía con respecto a su núcleo familiar. El deseo de formar una propia familia, de realzar un ámbito demasiado exaltado, podría dar cuenta de un deseo de ser madre a corta edad. Si bien no hay discursos explícitos al respecto, se observa en las entrevistadas quienes interrumpen sus estudios secundarios poca descripción de su entorno familiar (y de atributos positivos como en el caso de las madres que optan por continuar escolarizadas) o distanciamiento con su núcleo familiar. Esto no excluye u omite la situación de que la experiencia precoz de maternidad resulte muy difícil de sobrellevar para mujeres adolescentes, en especial porque no se cuenta ni con los recursos materiales ni con la disposición temporal y psicológica para asumirlo completamente. Tampoco de este análisis conviene realizar una generalización del tipo "todas las madres adolescentes que no completan estudios secundarios tienen un proyecto de vida asociado al proyecto familiar" o tampoco se podría advertir "todas las madres adolescentes escolarizadas no tienen en su proyecto de vida incorporada la dimensión familiar". Esas afirmaciones serían reduccionistas y supondrían cierta mecánica para el actuar humano, cuestión que las ciencias sociales han claramente descartado.

Estos modelos ideales nos permiten comprender un mapa diferenciado de los proyectos de vida de las madres adolescentes, si bien son una reducción analítica, 
dejan ordenar o iluminar ciertas zonas de la vivencia humana. Lo importante de reforzar es que cualquiera que sea el proyecto de vida de las madres adolescentes, una intervención a favor de ellas y su descendencia debiera considerar este tópico, central en la formación de identidad personal.

\subsection{Capital social de las madres adolescentes: debilitamiento de pares, preeminencia familiar}

Dado que en la socialización secundaria los pares juegan un rol significativo, en tanto promueven la integración social, se podría afirmar que, en esta etapa, el impacto biográfico de la maternidad no solo es significativo por el nuevo rol que se asume, que obliga a una "adultización" de la adolescencia, sino también por el desapego a un círculo de pares que constituye un referente social para la interacción social.

En este sentido, otra conclusión del estudio es que, si bien en adolescentes madres el capital social se debilita, la permanencia en el sistema escolar activa mecanismos de integración social que pueden favorecerlas al largo plazo. Por el contrario, quienes se desvinculan a la dinámica escolar entran en procesos de aislamiento social mucho mayor, abriendo aún más una brecha de menor identificación con el espacio escolar.

Esto se refuerza cuando Bourdieu (2000) nos señala: "el capital social está constituido por la totalidad de los recursos potenciales o actuales asociados a la posesión de una red duradera de relaciones más o menos institucionalizadas de conocimiento y reconocimiento mutuos" (p. 148). Esto significa que privarse de la esfera escolar es privarse de una red de conocidos, de contactos, de información, de proyectos, de espacios de participación activa, entre otros ámbitos.

Tanto adolescentes madres que abandonan la secundaria como las que permanecen en ella sí marcan estilos de comunicación limitados y vínculos con un número reducido de personas, pueden caer en el mismo riesgo de debilitamiento del capital social. Bourdieu (2000) nos advierte que la red de relaciones es el producto de estrategias individuales o colectivas de inversión, conscientes o inconscientemente dirigidas a establecer y mantener relaciones sociales que prometan más tarde o más temprano, un provecho inmediato. Por tanto, es necesario que en el campo de la escuela se promuevan espacios de participación y vinculación social de las embarazadas y las madres adolescentes. Esto porque el capital social solo puede existir sobre la base de relaciones de intercambio materiales y/o simbólicas permanentes. No basta con el deseo o la intención de relacionarse con otro, es preciso 
incidir en la cotidiana experiencia escolar, propiciando espacios de vinculación. Estos pueden ser desde las estrategias de aprendizajes (impulsando metodologías como trabajo cooperativo, aprendizaje-servicio, etc.), hasta actividades extra curriculares. Lo importante es que la escuela tiene un espacio donde puede actuar $y$, de acuerdo con lo señalado por las entrevistadas, no se está practicando.

La magnitud del capital social que posea un individuo depende de la extensión de la red de conexiones que este pueda efectivamente movilizar, como del volumen de capital (lo que transita en el intercambio: económico, cultural o simbólico). Al menos tres fuentes de capital social no institucionalizado revelan las entrevistadas: vínculos de pareja, vínculos familiares y, en menor medida, vínculo con amigos o pares.

En el caso de las madres adolescentes, se observa cierta asociación entre su decisión sobre el proceso de escolarización y el capital poseído. De esta manera, quienes optan por mantenerse en el sistema escolar son quienes poseen redes familiares más sólidas, que facilitan el cuidado del hijo o la hija, entendido no solo como apoyo para la crianza del niño o la niña, sino también como apoyo para la continuidad de su proceso educativo. Además, se trata de jóvenes que han pensado en la idea de un lugar institucionalizado de cuidado (jardín infantil) y han tenido la posibilidad de optar entre alternativas. En este caso, se vuelve explicito que el capital social ejerce un efecto multiplicador sobre el capital disponible, ya que facilita la continuidad de los estudios, aspecto que incrementa el capital cultural, amplía redes, lo que aumenta el capital social y a largo plazo puede traducirse en una mejor inserción laboral, lo que mejorará el capital económico. En otras palabras, poseen una red de conexiones para dar solución a ciertas problemáticas. En el caso de las madres que dejan la secundaria, la red de conexiones es menor, ya que se debilita con su salida del establecimiento.

\subsection{Campo: escuela, clave para el curso de acción educativo}

Para Bourdieu (2000), un campo es una configuración de relaciones objetivas donde se establecen posiciones entre los sujetos, agentes o instituciones según el poder (o capitales) que manejen. En el campo se establecen beneficios e intercambios de capital, así como relaciones con otras posiciones (dominación, subordinación, homología, etc.). El autor y sus seguidores han caracterizado ciertas normas de funcionamiento de los campos, una de ellas es que "en cualquier campo encontraremos una lucha, cuyas formas especificas habría que buscar cada vez, entre el recién llegado que trata de romper los cerrojos del derecho de 
entrada, y el dominante que trata de defender su monopolio y de excluir a la competencia" (p. 120).

En ese sentido, sostenemos que la escuela es un campo, caracterizado no solo por estructurarse con una jerarquía donde el poder lo ocupan "los que saben", "los encargados" (es decir, profesores, directivos y orientadores). En ese campo se ponen las reglas por lo general unilateralmente, es decir, no considerando necesariamente la opinión de los jóvenes. Aquí, se estructura además un currículo oculto, donde prácticas de estigmatización y discriminación de género cobran fuerza, ejemplo de ello es que la aceptación de madres adolescentes no es constante en todos los establecimientos de secundaria, tal como lo identifican Olavarría et al. (2007), de los doce mil establecimientos subvencionados en todos los niveles existentes en Chile, 2.461 matricularon alguna madre adolescente, no obstante suele observa una concentración en 7, que alberga un alta matrícula (superior a 100 casos) de madrea adolescentes.

Giddens (1999) sugiere que la escuela en este mundo globalizado y desbocado se ha vuelto una institución concha, impermeable a los cambios que demandan los nuevos tiempos. Podría ser una interpretación razonable. Junto a ella, otra interpretación puede estar en la percepción que tienen las entrevistadas, quienes sienten que el medio escolar desarrolla prejuicios de género, de clase y adulto-céntricos, elementos que pueden ser abordados desde una pedagogía de la igualdad que promueva procesos educativos incluyentes, abiertos a la diversidad y a la pluralidad cultural y social (Conde, 2014).

Si bien no se consultó explícitamente sobre posibles acciones discriminatorias, sí se hizo por los apoyos escolares. Se observa que las madres adolescentes que continúan escolarizadas no tienen de forma general, permanente e integral, facilitadores por parte de la institución escolar que contribuyan a su permanencia en el sistema. Lo que más demandan las entrevistadas es: Comprensión y Facilidades académicas.

La comprensión es entendida como el establecimiento de relaciones interpersonales y una red en el liceo, así como también el debilitamiento de prejuicios sociales y de género, así como discriminación en el liceo.

Resultan muy reveladoras las expectativas de las entrevistadas con respecto a mantener una buena relación con los docentes, esto es coherente con otros estudios que buscan entender qué puede estar cimentando la posibilidad de abandonar los estudios, cuando esta relación es valorizada en primer lugar, seguida de la relación con las y los compañeros, develando la importancia de las relaciones interpersonales (Cordero, 2008). 
En los casos en los que se advierten y valoran estos apoyos, se trata de concesiones explícitas y acotadas (tiempo limitado pre y posnatal o aceptación de inasistencia o retraso por asistencia al médico del hijo en algunas oportunidades más esporádicas). La realidad es que ocurren embarazos y las escuelas no están preparadas para asumirlos. Las medidas por considerar dependen de la voluntad personal de profesores y directivos, más que de procedimientos normados que regulen más claramente las reglas de juego y/o una cultura educativa de integración social. Por ello, cualquier intervención educativa a favor de la retención escolar debiera, al estudiar la trayectoria escolar de las adolescentes que son madres, reflexionar e identificar en la biografía escolar, en algunos hitos - acciones escolares (o capitales institucionales) que pudieran desincentivar la conclusión del ciclo educativo.

En ese sentido, no hay que olvidar que:

los estudiantes que tienen una alta autoestima aprenden más rápido, retienen la información por más tiempo, responden positivamente a los desafíos, poseen un grado de aceptación mayor frente a los demás y son capaces de responsabilizarse de sus actos, mientras que las personas que tienen un bajo concepto de sí mismas, tenderían a dejar más tempranamente el colegio, se volcarían a las drogas y demostrarían una variedad de patrones de comportamiento defensivo. (Saffie, 2000, p. 34)

Por lo que no solo es preciso restringir posibles estigmatizaciones o acciones discriminadoras (ya sea por patrones patriarcales de género, por la situación socioeconómica u otra), sino y, sobre todo, incrementar aquellas que aportan elementos de autoestima y reconocimiento en las madres adolescentes. La invitación es a pensar una nueva escuela, fundada sobre cimientos de inclusión de todos aquellos que tienen dificultades para un proceso de aprendizaje "estandarizado", una escuela que vea en cada escolar un sujeto y no un objeto, una escuela capaz de adaptarse a la diversidad de estudiantes. Una sistematización reciente señala que cuando las escuelas se piensan así mismas como mecanismos generadores de igualdad y se centran en la promoción de aprendizajes significativos, emerge el tema de la inclusión socioeducativa. Algunos sentidos que envuelve la noción de igualdad son: i) abordar problemáticas sociales, ii) valorar la pluralidad y la diferencia, iii) integrar a los alumnos con necesidades especiales, iv) re-pensar el currículo y el proceso de enseñanza-aprendizaje, v) identificar los diversos sentidos en la construcción de la infancia y la juventud (Vitar, 2008). 


\subsection{Estigmatización e identidad}

De las entrevistas se desprende que las adolescentes consultadas inician un proceso de aislamiento social y ruptura de vínculos con pares, al mismo tiempo que resienten las críticas basadas en prejuicios de género, al no responder a los modelos tradicionales esperados, y enfrentarse a su nueva condición social de madres, que además desean estudiar. Esta situación expresa prácticas de violencia al interior del establecimiento, las cuales son legitimadas en tanto no son cuestionadas. Como consecuencia, puede transformar su identidad personal no solo con respecto a la mirada sobre sí misma, sino también su relación con los demás. Ello es consistente con el hallazgo del estudio de Artavia (2013) que pone de manifiesto la violencia en espacios formales e informales de las dinámicas escolares; como, por ejemplo, desde los recreos hasta las relaciones interpersonales. En tal sentido, es muy conveniente recordar lo acuñado por Goffman (1995) cuando dice "carente de la saludable realimentación (feed-back) del intercambio social cotidiano con los demás, la persona que se aísla puede volverse desconfiada, depresiva, hostil, ansiosa y aturdida" (p. 24).

A ello se suma el surgimiento de un sentimiento de inferioridad de parte de las entrevistadas en relación con sus pares y las expectativas de familiares y el medio, lo que redunda en una visión crítica de sí mismas. En este sentido, propiciar intervenciones socioeducativas de acompañamiento de madres adolescentes permitiría mejorar su autoestima y aceptación. Goffman (1995) nos recuerda que

tener conciencia de la inferioridad significa que uno no puede dejar de formularse conscientemente cierto sentimiento crónico del peor tipo de inseguridad, y eso trae como consecuencia ansiedad (...) Ahora bien, esto representa una deficiencia casi fatal en el sistema del yo, ya que este no puede ocultar ni excluir una formulación definida: Soy inferior. Por lo tanto, la gente me tendrá aversión y yo no me sentiré seguro con ellos. (p. 25)

Siguiendo estas líneas, las consecuencias de la exposición al rechazo social o estigmatización por parte de las madres escolares es insospechado. No solo podrían incidir en su propia identidad, sino también en la relación que establecen con su hijo o hija, su futura pareja, sus familiares, amigos, futuros empleadores, etc. Cuestión que amerita un estudio de corte psicológico, para mayor comprensión de la situación. 
Asimismo, lo curioso es que la escuela, siendo el lugar idealizado para superar las desigualdades y abordar con equidad el desafío nacional de incrementar el capital humano, puede llegar a hacer todo lo contrario con las madres adolescentes, pues la incidencia de visiones y prácticas estigmatizadoras pueden debilitar el proceso educativo y la identidad de las madres. En otras palabras, las estigmatizaciones podrían desencadenar un movimiento centrífugo de las madres adolescentes, orientado hacia la expulsión de estas estudiantes, ya sea formal, bajo la manera de abandono escolar, o informal, como aislamiento social.

\section{Conclusiones y recomendaciones para programas públicos}

Las principales conclusiones son:

a) La decisión respecto a la maternidad, así como a la continuidad de estudios, se vincula al habitus de las madres adolescente, transmitido por sus familias.

b) La maternidad no es por sí mismo un factor que propicie, de forma causal y directa, el fracaso escolar. Hay otros factores concomitantes, latentes que, al yuxtaponerse con este cambio biográfico significativo, pueden generar como consecuencia el abandono escolar.

c) Varios caminos pueden tomar la trayectoria escolar de las madres adolescentes, las situaciones típicas identificadas son: i) deserción definitiva, donde se abandonan los estudios para toda la vida, ii) abandono transitorio, donde se interrumpen los estudios, re-integrándose con mucho tiempo de posterioridad, iii) abandono momentáneo, equivalente a lo que las madres trabajadoras tienen como derecho laboral (post natal), iv) continuar el proceso de escolarización, terminando el ciclo interrumpido, que generalmente es educación secundaria.

d) La maternidad adolescente marca un antes y un después en la biografía del adolescente y por consiguiente en su proyecto de vida.

e) Respecto al entorno social, la maternidad, no solo es significativo por el nuevo rol que se asume, que obliga a una adultización de la adolescencia, sino también por el desapego a un círculo de pares que constituye un referente social para la interacción social. Su capital social se debilita. De ahí la importancia de permanencia en el sistema escolar, que favorece mecanismos de integración social.

f) La escuela es un campo, caracterizado por estructurarse por una jerarquía administrativa basada en el poder del conocimiento. En ese campo se ponen las reglas por lo general unilateralmente, es decir, no considerando necesariamente la opinión de 
los jóvenes. En ese campo, se estructura además un currículo oculto, donde prácticas de estigmatización, discriminación y rechazo a situacionales especiales o no esperadas.

Del estudio se desprenden recomendaciones para las políticas públicas educativas que promuevan la inclusión, a través de programas socioeducativos corte preventivo o inclusivoreparador:

a) Con respecto a los programas preventivos, no basta con difundir la información sobre métodos anticonceptivos para disminuir los embarazos no planificados, es necesario: i) un enfoque que considere el capital cultural que tienen los adolescentes, aborde el desarrollo de proyectos de vida; ii) es necesario una articulación entre al menos dos sectores: salud y educación, de manera que la información y diálogo sobre la sexualidad y los proyectos de vida tengan su contraparte en el aumento de consultas de salud adolescentes.

b) En relación a programas inclusivos-reparadores, es necesario considerar: i) el enfoque a favor de la permanencia y no solo anti-deserción. Ello sitúa el foco de atención en una acción integral donde todos los actores tienen una responsabilidad; lo que implica trabajar con las madres y padres adolecentes, con los profesores, directivos y orientadores, para ahondar en el campo (escolar); ii) abordarse también temas que apoyen la reconstrucción biográfica de las madres adolescentes y el fortalecimiento de proyecto de vida; iii) propiciar escuelas inclusivas, donde en el currículo oculto no primen visiones ni prácticas estigmatizadoras y discriminatorias de género, etnia, nivel socio económico, iv) promover la resiliencia en la escuela (Melillo, 2003), entendiendo ésta como la capacidad de los individuos de sobreponerse a las experiencias negativas y adaptarse con éxito frente a la adversidad, Henderson y Milstein (2003) señalan que: "la disposición a emprender acciones para abordar, comprender y reforzar el desarrollo de la resiliencia está surgiendo no sólo entre los científicos sociales sino también entre los docentes que comienzan a percibir la necesidad de que las escuelas sean instituciones que fomenten la resiliencia para aquellos que trabajan y estudian en ellas" (p.45).

c) Es loable una articulación intersectorial que favorezca la entrega de un grupo beneficios de soporte para las madres adolescentes, entre ellas: i) sector educación, nivel pre escolar público (JUNJI e INTEGRA): ya sea con jardín infantil exclusivo para madres adolescentes por comuna o integrando a los jardines tradicionales, con un trato 
especial para madres adolescentes, que permita que puedan tener la tranquilidad por un adecuado cuidado de sus hijos y por tanto disponer de tiempo para la asistencia escolar; ii) sector salud: realizando una permanente atención de salud de los hijos de las madres adolescentes, a través del Programa "Control del niño de sano", que se desarrolló en los centros de salud primaria en Chile, favorecería monitorear el bienestar de los niños y apoyar el rol protector de la madre adolescente. Además, podrían realizarse procesos de capacitación sobre pautas de crianza y desarrollo integral del niño, para brindar más herramientas a las adolescentes para ejercer su rol parental, y , iii) sector desarrollo integral de los niños y jóvenes (Junta Nacional de Auxilio Escolar y Becas -JUNAEB-: favorecer la entrega de becas, alimentación, apoyo en locomoción pública, útiles escolares a las madres adolescentes, de manera que no tengan limitaciones para asistir al establecimiento escolar, en caso de no contar los recursos para su traslado, alimentación durante la jornada escolar, y acceso a útiles escolares.

\section{Referencias}

Alvarado, Kathia. (2005). Concepciones acerca de la maternidad en la educación formal y no formal. Revista Electrónica Actualidades Investigativas en Educación, 5(1), 1-27. Recuperado de http://revistas.ucr.ac.cr/index.php/aie/article/view/9117/17499

Araya Umaña, Sandra. (2004). Hacia una educación sexista. Revista Electrónica Actualidades Investigativas en Educación, 4(2), 1-14. Recuperado de http://www.redalyc.org/pdf/447/44740217.pdf

Artavia, Jenny. (2013). Manifestaciones ocultas de violencia, durante el desarrollo del recreo escolar. Revista Electrónica Actualidades Investigativas en Educación, 13(1), 1-21. Recuperado de http://revista.inie.ucr.ac.cr/index.php/aie/article/view/599

Arguedas, Irma y Jiménez, Flor. (2007). Factores que promueven la permanencia de estudiantes en la educación secundaria. Revista Actualidades Investigativas en Educación, 7(3), 1-36. Recuperado de http://revista.inie.ucr.ac.cr/index.php/aie/article/view/191

Arriagada, Irma. (2003). Dimensiones de la pobreza y políticas desde una perspectiva de género. Quito, Ecuador: CEPAL. Recuperado de http://www.cepal.org/mujer/reuniones/quito/iarriagada2.pdf

Assef, Verónica. (1996). Embarazo Adolescente: Una realidad nacional. Santiago: CEANIM.

Bernstein, Basil. 1974. Códigos amplios y restringidos: sus orígenes sociales y algunas consecuencias. En Paul Garvin y Yolanda Lastra (Eds.), Antología de estudios de etnolingüística y sociolingüística, (pp. 357-374). México: UNAM.

Bourdieu, Pierre. (2000). Poder, Derecho y Clases sociales. España: Editorial Declei de Broker S.A. 
Canales, Manuel. (2006). Metodologías de investigación social. Introducción a los oficios. Chile: LOM ediciones.

Castillo, Mario y Gamboa, Ronny. (2013). La vinculación de la educación y género. Revista Electrónica Actualidades Investigativas en Educación, 13(1), 1-16. Recuperado de http://revista.inie.ucr.ac.cr/index.php/aie/article/view/613

Casassus, Juan. (2006). La educación del ser emocional. Chile: Editorial cuarto propio.

Comisión Económica para América Latina y el Caribe (CEPAL). (2008). Superar la pobreza mediante la inclusión social. Santiago de Chile: CEPAL.

Conde, Francisco Javier. (2014). Desigualdad, discriminación y pedagogía de la igualdad. Revista Electrónica Actualidades Investigativas en Educación, 14(1), 1-20. Recuperado de http://revista.inie.ucr.ac.cr/index.php/aie/article/view/679

Cordero, Teresita. (2008). Opinión de un grupo de docentes sobre la deserción Escolar. Explorando sobre sus actuaciones en el Contexto institucional. Revista Actualidades Investigativas en Educación, 8(3), 1-33. Recuperado de http://revista.inie.ucr.ac.cr/index.php/aie/article/view/233

García, Andrés. (2000). Introducción. En Pierre Bourdieu (Comp.), Poder, Derecho y Clases Sociales (pp. 9-60). España: Editorial Declei de Broker S.A.

Giddens, Anthony. (1999). Un mundo desbocado. España: Taurus.

Goffman, Erwing. (1995). Estigma. La identidad deteriorada. Argentina: Amorrortu Editores.

Gutiérrez, Claudia y Duarte, Klaudio. (2005). Estudio sobre las acciones públicas y recursos disponibles para adolescentes en relación al embarazo y paternidad/maternidad. Santiago: INAP, Universidad de Chile.

Hamel, Patricia. (1992). El Embarazo en Adolescentes. Santiago: Unicef.

Hamel, Patricia. (1994). Realidades y desafíos: Reflexiones de mujeres que trabajan en salud reproductiva. Santiago: ICMER.

Henderson, Nan y Milstein, Mike. (2003). Resiliencia en la escuela. Argentina: Paidós editores.

Instituto Nacional de la Juventud (INJUV). (2010). VI Encuesta Nacional de Juventud. Chile: el autor.

Martínez, Javier y Palacios, Margarita. (1996). Informe sobre la decencia. La diferenciación estamental de la pobreza y los subsidios públicos. Chile: Ediciones Sur.

Ministerio de Salud de Chile (Minsal). (2011). Estadísticas de natalidad y mortalidad. Chile: Departamento Estadísticas e Información de Salud, DEIS. 
Ministerio de Salud de Chile (Minsal). (2012). Situación actual del embarazo adolescente en Chile. Chile: Subsecretaría de Salud Pública. División y Control de Enfermedades Depto. de Ciclo Vital. Programa Nacional de Salud Integral de Adolescentes y Jóvenes. Chile.

Mella, Orlando. (2003). Metodología cualitativa en ciencias sociales y educación. Chile: Editorial Primus.

Melillo, Aldo. (2003). Resiliencia y educación. En Aldo Melillo, y Elbio Suárez (Comps.), Resiliencia. Descubriendo las propias fortalezas (pp. 123-144). Buenos Aires: Paidos.

Molina, Ramiro, Molina, Temístocles y González, Electra. (2007). Madres niñas-adolescentes de 14 años y menos. Un grave problema de salud pública no resuelto en Chile. Revista Médica de Chile, 135(1), 79-86.

Núñez, Lorena; Segall, Marcela; Vivanco, Sergio. (2002). Análisis y detección de expectativas y proyecto de vida de niñas, niños y adolescentes (Documento de Trabajo $\left.N^{\circ} 80\right)$. Santiago: SERNAM.

Olavarría, José, Palma, Julieta, Donoso, Alina, Valdés, Teresa y Olivera, Patricio. (2007). Estudio de la situación de maternidad y paternidad en el sistema educacional chileno. Santiago: Ministerio de Educación de Chile -Cedem.

Palma, Irma y Quilodrán, Cecilia. (1992). Embarazo adolescente: desde el matrimonio al aborto, respuestas en relación al proyecto de vida (Informe de investigación de la Escuela de Psicología de la Universidad de Chile, patrocinado por Fundación Carlos Chagas y Mac Arthur Foundation). Recuperado http://www.pasa.cl/wpcontent/uploads/2011/08/Informe de investigacion Fundacion Chagas Palma Irma Quilodran Ceci.doc

Reyes, Román (Dir.). (s.f.). Habitus. Diccionario Crítico de Ciencias Sociales. Recuperado de www.ucm.es/info/eurotheo/diccionario/H/habitus.htm

Ritzer, George. (1993). Teoría Sociológica Contemporánea. México: Mc Graw Hill.

Rodríguez, Jorge. (agosto, 2005). Reproducción en la adolescencia: el caso de Chile y sus implicaciones de política. Revista de la CEPAL, (86), 123-146.

Sadler, Michele y Aguayo, Francisco. (2007). Gestación adolescente y dinámicas familiares. Estudio de las Dinámicas familiares en familias de padres y madres adolescentes. Santiago: Mideplan/Fosis, CIEG-Universidad de Chile.

Saffie, Nubia. (2000). Valgo o no valgo. Autoestima y rendimiento escolar. Santiago: LOM Ediciones.

Tenti, Emilio. (2000). Socialización. Buenos Aires, Argentina: IIPPE-UNESCO. Recuperado http://www.buenosaires.iipe.unesco.org/documentos/socializaci-n

Touraine, Alain. (2006). ¿Podremos vivir juntos? México: Fondo de Cultura Económica.

Vitar, Ana. (2008). "Escuelas que hacen inclusión". En Marcelo Krichesky (Comp.), Adolescencia e inclusión educativa. Un derecho en cuestión (pp. 47-62). Argentina: Ediciones NOVEDUC - OEI - UNICEF - Fundación SES. 